\title{
Tripeptide tyroserleutide plus doxorubicin: therapeutic synergy and side effect attenuation
}

\author{
Zhi-feng Zhu ${ }^{\dagger 1}$, Li-juan Chen ${ }^{\dagger 1}$, Rong Lu ${ }^{1,2}$, Jing Jia ${ }^{1}$, Yu Liang ${ }^{3}$, Qiong Xu ${ }^{1}$, \\ Chun-lei Zhou ${ }^{1}$, Li Wang ${ }^{1}$, Song Wang ${ }^{1}$ and Zhi Yao*1,4
}

\begin{abstract}
Address: ${ }^{1}$ Department of Immunology, Tianjin Medical University, Tianjin 300070, PR China, ${ }^{2}$ Shenzhen Kangzhe Pharmaceutical Co. Ltd., Shenzhen 518057, PR China, ${ }^{3}$ Department of Histology and Embryology, Tianjin Medical University, Tianjin 300070, PR China and ${ }^{4}$ Tianjin Key Laboratory of Cellular and Molecular Immunology, Tianjin Medical University, Tianjin 300070, PR China

Email: Zhi-feng Zhu - zhuzhifengen@yahoo.com.cn; Li-juan Chen - mingiiao8002@yahoo.com.cn; Rong Lu - rong627@yahoo.com; Jing Jia - jiajing09@yahoo.com.cn; Yu Liang - liangy@tijmu.edu.cn; Qiong Xu - xvqiong1980@yahoo.com.cn; Chun-

lei Zhou - zhou_chun_l@yahoo.com.cn; Li Wang - wlee7923@yahoo.com.cn; Song Wang -wangsong_1979@yahoo.com.cn; Zhi Yao* - yaozhi@tmu.cn

* Corresponding author †Equal contributors
\end{abstract}

Published: 25 November 2008

BMC Cancer 2008, 8:342 doi:|0.1|86/|47|-2407-8-342
Received: 4 January 2008

Accepted: 25 November 2008

This article is available from: http://www.biomedcentral.com//47I-2407/8/342

(C) 2008 Zhu et al; licensee BioMed Central Ltd.

This is an Open Access article distributed under the terms of the Creative Commons Attribution License (http://creativecommons.org/licenses/by/2.0), which permits unrestricted use, distribution, and reproduction in any medium, provided the original work is properly cited.

\begin{abstract}
Background: Tripeptide tyroserleutide (YSL) is a novel small molecule anti-tumor polypeptide that has been shown to inhibit the growth of human liver cancer cells. In this study, we investigated the effects of YSL plus doxorubicin on the growth of human hepatocellular carcinoma BEL-7402 cells that had been transplanted into nude mice.
\end{abstract}

Methods: Nude mice bearing human hepatocellular carcinoma BEL-7402 tumors were treated with successive intraperitoneal injections of saline; low-, mid-, or high-dose doxorubicin; or low-, mid-, or high-dose doxorubicin plus YSL. Effects on the weight and volume of the tumors were evaluated.

Results: Co-administration of YSL and high-dose doxorubicin $(6 \mathrm{mg} / \mathrm{kg}$ every other day) prolonged the survival time of tumor-bearing mice as compared to high-dose doxorubicin alone. As well, the anti-tumor effects of mid- and low-dose doxorubicin (2 and $0.7 \mathrm{mg} / \mathrm{kg}$ every other day, respectively) were enhanced when supplemented with YSL; the tumor growth inhibition rates for YSL plus doxorubicin were greater than the inhibition rates for the same dosages of doxorubicin alone. The combination of YSL and doxorubicin decreased chemotherapy-associated weight loss, leukocyte depression, and heart, liver, and kidney damage as compared to doxorubicin alone.

Conclusion: The combination of YSL plus doxorubicin enhances the anti-tumor effect and reduces the side effects associated with doxorubicin chemotherapy.

\section{Background}

According to the World Health Organization, 7.6 million people succumbed to cancer in 2005, accounting for $13 \%$ of all deaths. However, in China, the cancer death rate was $20.2 \%$, and it is expected to reach $23.6 \%$ by 2023 . Liver cancer is the third most common cause of death in China [1].

Anthracene-nucleus antibiotics are anti-tumor drugs that have been heavily researched and developed for the past 
twenty years. Among those, doxorubicin is the most commonly used and the most important; however, its use has been increasingly limited due to severe associated side effects, such as myocardial toxicity and bone marrow depression [2]. Because the dosage of doxorubicin can be decreased when doxorubicin is given combination with other anti-tumor drugs, which can also elevate the therapeutic effect and relieve side effects of doxorubicin, combination therapy has become a common strategy for doxorubicin-based chemotherapy.

Tripeptide tyroserleutide (YSL) is a small molecule polypeptide that consists of three natural amino acids: Ltyrosine, L-serine, and L-leucine. In our laboratory, we have observed anti-tumor activity for YSL both in vitro and in vivo using the MTT method, the mouse model of ascites fluid-type hepatocarcinoma $\mathrm{H} 22$, and the nude mouse model of human hepatocellular carcinoma $[3,4]$. In this study, we established a nude mouse model of human hepatocellular carcinoma in order to investigate the effect of YSL on tumor growth when given in combination with doxorubicin.

\section{Methods \\ Cell culture}

Human hepatocellular carcinoma BEL-7402 cells were purchased from Institute of Cell Biology, Shanghai, Academia Sinica. The cells were cultured in RPMI 1640 supplemented with $10 \%$ fetal bovine serum in a humidified atmosphere of $5 \% \mathrm{CO}_{2}$ at $37^{\circ} \mathrm{C}$. During cultivation, BEL-7402 cells remained free of mycoplasma or bacterial contamination.

\section{Animals}

Healthy female BALB/c (nu/nu) mice (4-5 weeks old, 18$22 \mathrm{~g}$ ) were obtained from Chinese Academy of Medical Sciences (Beijing, China). The animals were housed at our university under specific pathogen-free conditions according to the guidelines of the Chinese Association for Laboratory Animal Care, using a laminar airflow rack. Animals had continuous access to sterilized food pellets and autoclaved water, and a 12-hr light/dark cycle. The temperature was maintained at $22-26^{\circ} \mathrm{C}$ and $50-70 \%$ relative humidity. All studies were performed according to the regulations of Tianjin Medical University experimental animal center and obtained the ethical approval from Tianjin Council for the Care of Animal in Medical Research.

\section{Nude mouse model of human hepatocellular carcinoma} [5]

To establish the human hepatocellular carcinoma model, BEL-7402 cells in exponential phase were grown to a density of $1 \times 10^{8} / \mathrm{ml}$. $0.1 \mathrm{ml}$ of the cell suspension was injected subcutaneously into the right back of every nude mouse. Mice with tumors of uniform volume were selected and randomly divided into different treatment groups when the tumor volume reached $100 \mathrm{~mm}^{3}$.

\section{YSL plus high-dose doxorubicin combination therapy}

YSL was obtained from Shenzhen Kangzhe Pharmaceutical, China. Doxorubicin (Adriamycin, ADM) was obtained from Zhejiang Haizheng Pharmaceutical Co., Ltd.

Nude mice bearing tumors were randomly divided into high-dose doxorubicin ( $6 \mathrm{mg} / \mathrm{kg}$ every other day), YSL $(320 \mu \mathrm{g} / \mathrm{kg} / \mathrm{d})$, YSL $(320 \mu \mathrm{g} / \mathrm{kg} / \mathrm{d})$ plus high-dose doxorubicin (6 mg/kg every other day), and saline $(0.2 \mathrm{ml} / \mathrm{d})$ groups; each group had twenty mice. All medicines were dissolved in $0.2 \mathrm{ml}$ saline. Doxorubicin was administered intraperitoneally (IP) every other day, YSL and saline (control) were administered every day. For animals receiving combination therapy, the two medications were administered at a 4-hr interval. Mice received successive IP administrations for 30 days or until the death of mouse. Beginning on the day after the first administration, we observed activity, stool color and stool patterns of the mice in all treatment groups. We recorded the survival status of mice daily and calculated the rate of life extension according to the following formula: the rate of life extension $=$ (average survival days of experiment group-average survival days of control group)/average survival days of control group $\times 100 \%$.

\section{YSL plus mid-dose doxorubicin combination therapy}

Nude mice bearing tumors were randomly divided into mid-dose doxorubicin ( $2 \mathrm{mg} / \mathrm{kg}$ every other day), YSL $(320 \mu \mathrm{g} / \mathrm{kg} / \mathrm{d})$, YSL $(320 \mu \mathrm{g} / \mathrm{kg} / \mathrm{d})$ plus mid-dose doxorubicin (2 mg/kg every other day), and saline $(0.2 \mathrm{ml} / \mathrm{d})$ groups; each group had twelve mice. Mice received IP administrations for 30 successive days. The weights of mice were measured every three days until the experiment was terminated.

On the day after treatment termination, blood samples were obtained from the angular vein and anticoagulated with heparin. We measured hemoglobin levels, blood platelets count, and leukocyte counts. Hearts, livers, and kidneys were fixed immediately in $10 \%$ formalin. Paraffin-embedded sections of each tissue sample were stained with hematoxylin and eosin (HE). The weight and three perpendicular diameters of every tumor were recorded. Tumor volume was calculated by the following formula: $\mathrm{V}$ $=(1 / 6) \pi \mathrm{ABC}$, where $\mathrm{V}$ is the tumor volume, and $\mathrm{A}, \mathrm{B}$, and $\mathrm{C}$ are the three tumor diameters. The rate of tumor inhibition $=$ (average tumor weight of control group - average tumor weight of experiment group)/average tumor weight of control group $\times 100 \%$. 


\section{YSL plus low-dose doxorubicin}

Nude mice bearing tumors were randomly divided into low-dose doxorubicin $(0.7 \mathrm{mg} / \mathrm{kg}$ every other day), YSL $(320 \mu \mathrm{g} / \mathrm{kg} / \mathrm{d})$, YSL $(320 \mu \mathrm{g} / \mathrm{kg} / \mathrm{d})$ plus low-dose doxorubicin $(0.7 \mathrm{mg} / \mathrm{kg}$ every other day $)$, and saline $(0.2 \mathrm{ml} / \mathrm{d})$ groups; each group had twelve mice. Treatments were administered IP for 60 successive days. Mouse characteristics including body weights, blood counts, and tumor volumes and weights were observed and recorded as described in the study of mid-dose doxorubicin.

\section{Statistical Analysis}

Data are expressed as mean \pm standard deviation (SD). Statistical significances of survival time differences were analyzed according to the Kaplan-Meier method using SPSS software. The statistical significance of other differences was tested using one-way analysis of variance (ANOVA) followed by the Student-Newman-Keuls test. Statistical significance was set at $\mathrm{P}<0.05$.

\section{Results}

YSL plus high-dose doxorubicin prolongs the survival time of nude mice bearing tumors compared with doxorubicin alone

After about 10 days of administration, the mice that received high-dose doxorubicin alone showed weight loss and decreased activity level. Similar changes were observed in the YSL plus doxorubicin group; however, in general, the condition of the combination group was better than that of the doxorubicin group. No obvious side effects were observed in YSL group.

All the animals in YSL group and saline group were still alive 30 days later (termination of the experiments). The mean survival time of mice that received YSL plus highdose doxorubicin was longer than that of mice that received high-dose doxorubicin alone. This difference was significant, and the extension rate of survival time was 16.67\% $(P<0.05$; Figure 1).

\section{Co-administration of YSL diminishes the side effects associated with mid-dose doxorubicin}

After about 12 days, the mice treated with mid-dose doxorubicin alone showed decreased body temperature and lackluster hair. Similar changes were observed in the YSL combination group, but in general, their condition was better than that of the doxorubicin group. The body temperature of the mice that received YSL and doxorubicin was lower than that of the mice that received doxorubicin alone. Mice in YSL group and saline group were in good physical condition and showed weight gain. There were no significant difference in weight gain between YSL group and saline group. The weight of the mice in YSL plus mid-dose doxorubicin and mid-dose doxorubicin alone was lower after drug administration than before administration. However, the mean weight of the YSL combination group was higher than the mean weight of the doxorubicin group, and the mean weight loss for YSL combination group was lower than that of the doxorubicin group. As well, the weight loss in YSL combination group manifested later than in the doxorubicin group (Figure 2).

There were no significant difference in hemoglobin levels, blood platelets count and between YSL group and saline group. Leukocyte counts were markedly lower in the doxorubicin group than in the YSL combination group and YSL alone group, and were significantly lower as compared to the saline group $(\mathrm{P}<0.05)$. Blood platelet counts were higher in doxorubicin group than in both the YSL combination and saline groups, but there was no statistical significance versus the saline group $(\mathrm{P}>0.05)$. Hemoglobin levels in both the doxorubicin and YSL combination groups were higher than in the saline group, but these differences were not statistically significant (Figure 3).

Including YSL in the treatment regimen decreased the toxic effects of mid-dose doxorubicin on the heart, liver, and kidney of nude mice bearing tumors as shown by $\mathrm{HE}$ staining (Figure 4). In the mid-dose doxorubicin group, there was puffing of cardiac muscle cells, enhanced acidophilia, and augmented interspaces. The presence of these pathological changes was softened in the YSL combination group, in which the structure of heart muscle was comparatively complete. The structure of hepatic lobules was not clear in the mid-dose doxorubicin group, and there was enhanced acidophilia and interstitial edema. However, in the YSL combination group the structure of the hepatic lobules was clear. The structure of the kidney cortical labyrinth and medullary ray was acceptable in the mid-dose doxorubicin group, but the cell proliferation of the glomeruli decreased, there was party acinus renalis blood capillary glomus congestion, narrowed Bowman's space, and interstitial congestion. These effects were muted slightly in the YSL combination group. There are no obvious pathological changes in YSL alone and saline group.

YSL alone and mid-dose doxorubicin could inhibit tumor growth in nude mice bearing human hepatocellular carcinoma BEL-7402 tumors. The tumor inhibition rate was $34.39 \%$ and $53.35 \%$, respectively, which were statistically significant as compared with the saline group $(\mathrm{P}<0.05)$. The tumor inhibition rate for combination therapy was $56.15 \%$, which was statistically significant as compared to the saline group $(\mathrm{P}<0.05)$. However, there was no significance difference between the inhibition rates of the YSL combination group and the doxorubicin group $(\mathrm{P}>0.05$; Figure 5). 


\section{Survival Functions}

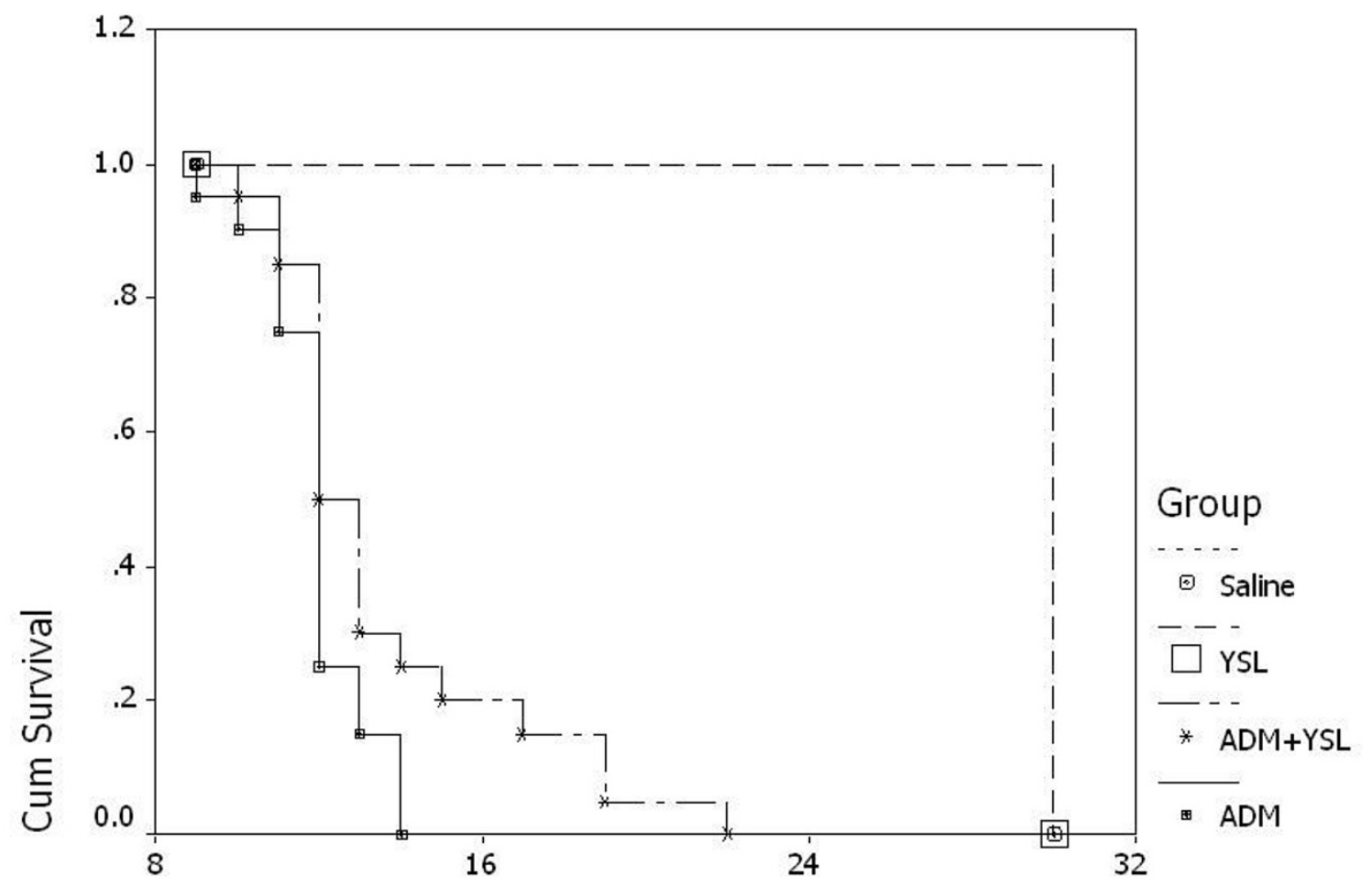

Survival time (d)

\section{Figure I}

Survival times of nude mice bearing human hepatocellular carcinoma BEL-7402 tumors treated with either high-dose doxorubicin or YSL plus high-dose doxorubicin. BEL-7402 cell suspension $\left(\mathrm{I} \times 10^{7} / \mathrm{each} \mathrm{mouse}\right) \mathrm{was}$ subcutaneously inoculated into the right back of every nude mouse. When tumor volume research $100 \mathrm{~mm}^{3}$, they were randomly divided into different groups $(n=20)$. The mice were administrated i.p. for 30 days or until the death of mouse. Statistical significances of survival time were tested by Kaplan-Meier method using SPSS, and difference between the group of high dosage of ADM and the group of YSL combination group was tested by Log Rank, statistical significance was set at $P<0.05$. ADM, doxorubicin or adriamycin; YSL, tripeptide tyroserleutide.

\section{The anti-tumor effects of YSL plus low-dose doxorubicin} Mice in four groups, including YSL group, YSL plus lowdose doxorubicin group, low-dose doxorubicin group and saline group, were in good physical condition and showed weight gain. Mice treated with YSL group or lowdose doxorubicin $(0.7 \mathrm{mg} / \mathrm{kg}$ every other day) or YSL plus low-dose doxorubicin tolerated therapy well, there were no overt side effects in either group. The mean body weight of nude mice in the YSL combination group was higher than that in doxorubicin group, but this difference was not significant $(P>0.05$; Figure 6$)$.
There were no obvious differences in white blood cell counts, platelet counts, or hemoglobin levels among the three treatment groups, and there were no significance differences as compared to the saline group $(\mathrm{P}>0.05$; Figure 7).

The groups treated with low-dose doxorubicin were observed for 60 successive days. The tumor inhibition rate of YSL alone group and YSL combination group were $37.11 \%$ and $52.17 \%$, respectively, higher than that of the low-dose doxorubicin group (30.14\%). The tumor inhibition rate of the YSL combination group was significantly 


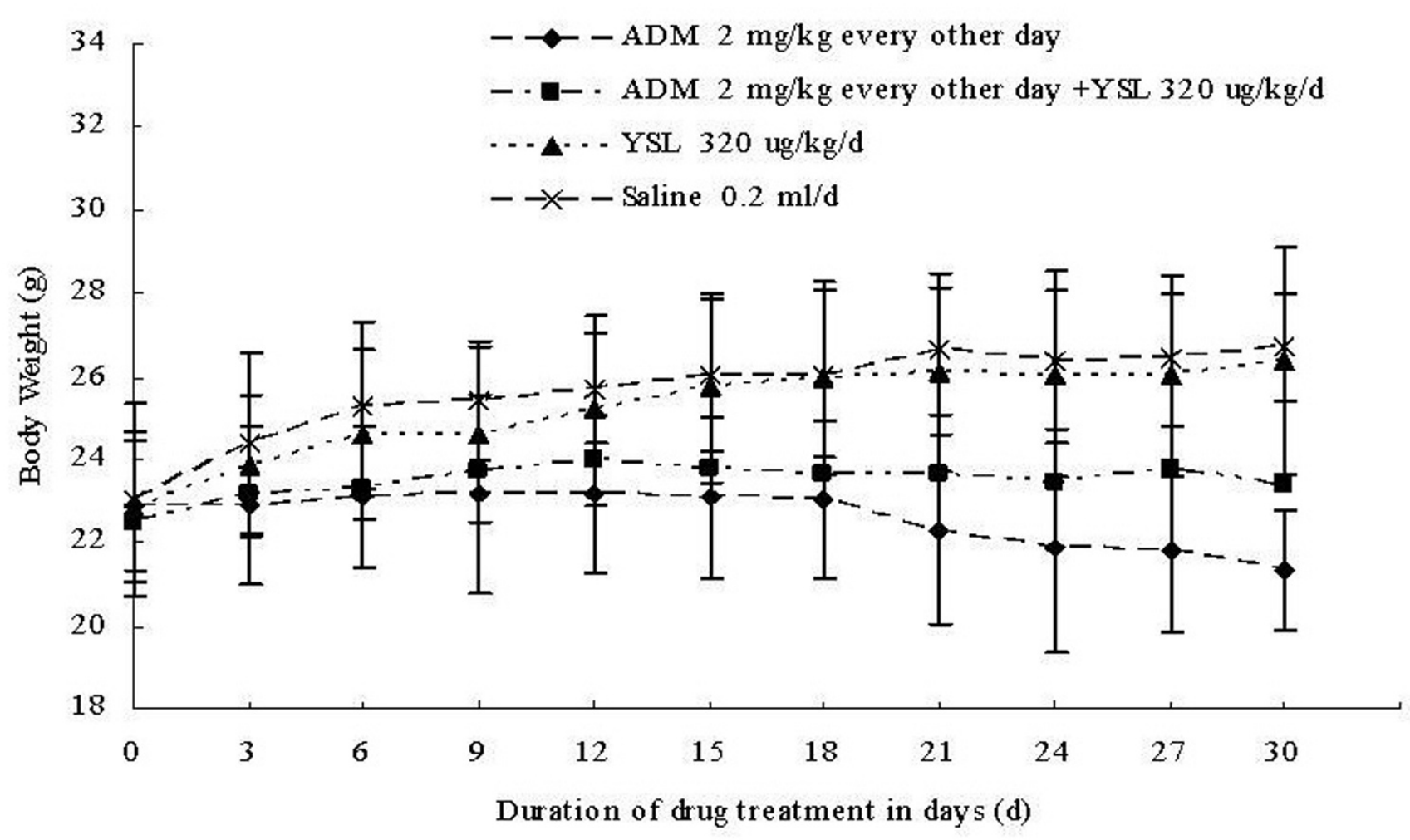

Figure 2

The effects of YSL plus mid-dose doxorubicin on the weight of nude mice bearing human hepatocellular carcinoma BEL-7402 tumors. BEL-7402 cell $(\mathrm{I} \times 107 /$ every mouse) was subcutaneously inoculated into the right back of every nude mouse. When the tumors had reached an average volume of $100 \mathrm{~mm}^{3}$, the tumor-bearing nude mice were randomly divided into different groups $(n=12)$. Drugs delivered by i.p. injection for 30 days successively. We weighted every group mice by electronic balance every 3 day and recorded the weight. Data points represent mean body weight \pm SD. ADM, doxorubicin or adriamycin; YSL, tripeptide tyroserleutide.

different as compared to the saline group ( $\mathrm{P}<0.05)$, but not as compared to the doxorubicin group $(\mathrm{P}>0.05$; Figure 8).

\section{Discussion}

Doxorubicin is a broad-spectrum antibiotic used widely for its anti-tumor activity. It is considered one of the most useful anti-tumor drugs available [6]. However, it's clinical use is limited by its serious side effects including bone marrow depression [7], liver and kidney injury [8,9], and cardiac toxicity [10]. Cardiac toxicity is considered the main side effect; some consider doxorubicin's dosedependent cardiac toxicity to be more dangerous than its other serious side effects [11]. When doxorubicin is combined with other chemotherapeutic agents such as cyclophosphamide, bleomycin, and cisplatin, doxorubicin's cardiac toxicity is amplified. Therefore, doxorubicin dosage cannot be escalated and its use in combination therapy is limited. Identifying an acceptable therapeutic partner for doxorubicin remains an area of considerable clinical interest.

YSL is novel anti-tumor peptide that can inhibit the growth of human hepatocellular carcinoma cells without observable side effects. High performance and low toxicity are characteristic of YSL, and have become the basis efforts to use YSL and doxorubicin combination therapy to cure hepatoma. In this study, we imitated the clinical conditions of doxorubicin therapy and examined the therapeutic efficacy of YSL in combination with different dosages of doxorubicin. We evaluated the effects on the survival in a nude mouse model of human hepatocellular carcinoma.

Changes in tumor volume and weight are the most direct indices for judging the efficacy of anti-tumor therapy. We found that the tumor inhibition rate was enhanced when YSL was combined with mid- or low-dose doxorubicin, demonstrating that YSL enhances the anti-tumor effect of 


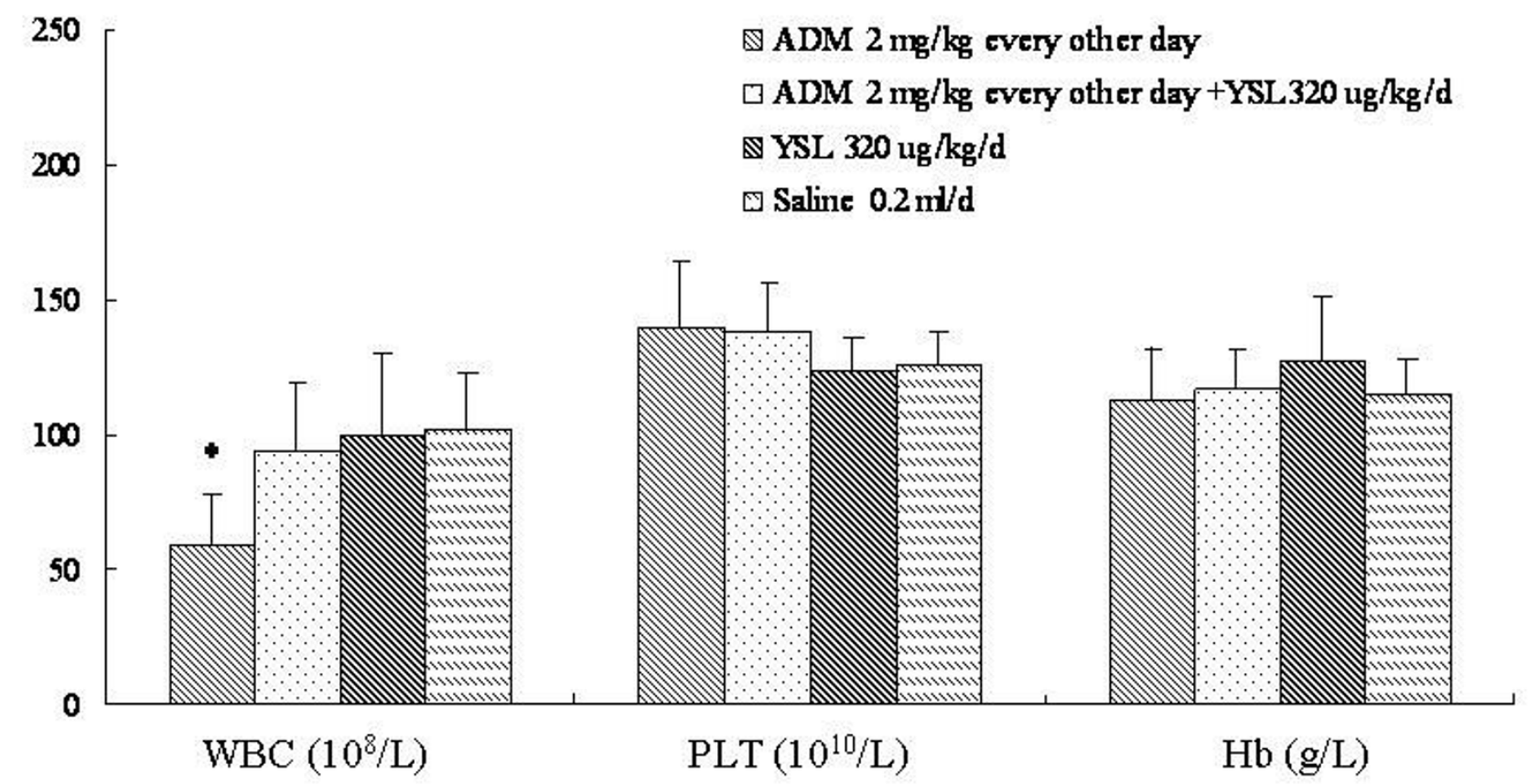

Figure 3

The effects of YSL plus mid-dose doxorubicin on the hematological indices of nude mice bearing human hepatocellular carcinoma BEL-7402 tumors. BEL-7402 cell $(\mathrm{I} \times 107 /$ every mouse) was subcutaneously inoculated into the right back of every nude mouse. When the tumors had reached an average volume of $100 \mathrm{~mm}^{3}$, the tumor-bearing nude mice were randomly divided into different groups $(n=12)$. Drugs delivered by i.p. injection for 30 days successively. At the next day of the last administration, blood was obtained from angular vein of nude mice, then blood was anticoagulated by heparin, we detected hematoglobin level and counted PLT and WBC. All data were analyzed by one-way analysis of variance using SPSS. The difference between two groups was analyzed by the Student-Newman-Keuls test. Standard deviation was represented by error line. $* P<0.05$ as compared to the saline group. ADM, doxorubicin or adriamycin; YSL, tripeptide tyroserleutide. WBC, white blood cells; PLT, platelets; Hb, hemoglobin.

doxorubicin. In particular, the tumor inhibition rate of YSL plus low-dose doxorubicin was higher than that of the doxorubicin alone, whereas there was no statistically significant difference between the doxorubicin and saline groups. The manifestation of therapeutic action when low-dose doxorubicin is given in conjunction with YSL versus no therapeutic action when given alone suggests that YSL synergizes with doxorubicin. The inclusion of YSL can reduce the dosage required to obtain the clinical benefit of doxorubicin while limiting the potential side effects. These data are consistent with the principle of combined therapy for tumor treatment [12].

Other key indices for evaluating tumor therapy efficacy are survival time and survival quality. Research about survival quality has received increasing attention in the international community in recent years. Survival quality (quality-of-Life, QOL) has been widely used as an index to evaluate therapeutic results $[13,14]$. At the 2005 meeting of the American Society of Clinical Oncology (ASCO), more than 98 articles about survival quality were presented. The completely new indexes-elevate survival quality and prolong survival time on tumor therapy curative effect was introduced clearly at ASCO 2007. In clinical anti-tumor therapy, elevating patient survival quality includes malignant tumor rehabilitation care [15], palliative treatment [16], and attenuation and synergy of radiotherapy and chemotherapy [17].

One focus of our research is on the attenuation and synergistic effects of YSL in combination chemotherapy, and the superiority of YSL for treating hepatoma. We first observed the attenuating effects of YSL in combination with deadly dosages of doxorubicin. High dosages of doxorubicin ( $6 \mathrm{mg} / \mathrm{kg}$ every other day) can induce death in mice; however, adding YSL to high-dose doxorubicin significantly prolongs the survival time of nude mice bearing tumors as compared to doxorubicin alone. These data suggest that YSL enhances nude mice's tolerance to doxorubicin, thereby increasing doxorubicin's clinical value. 

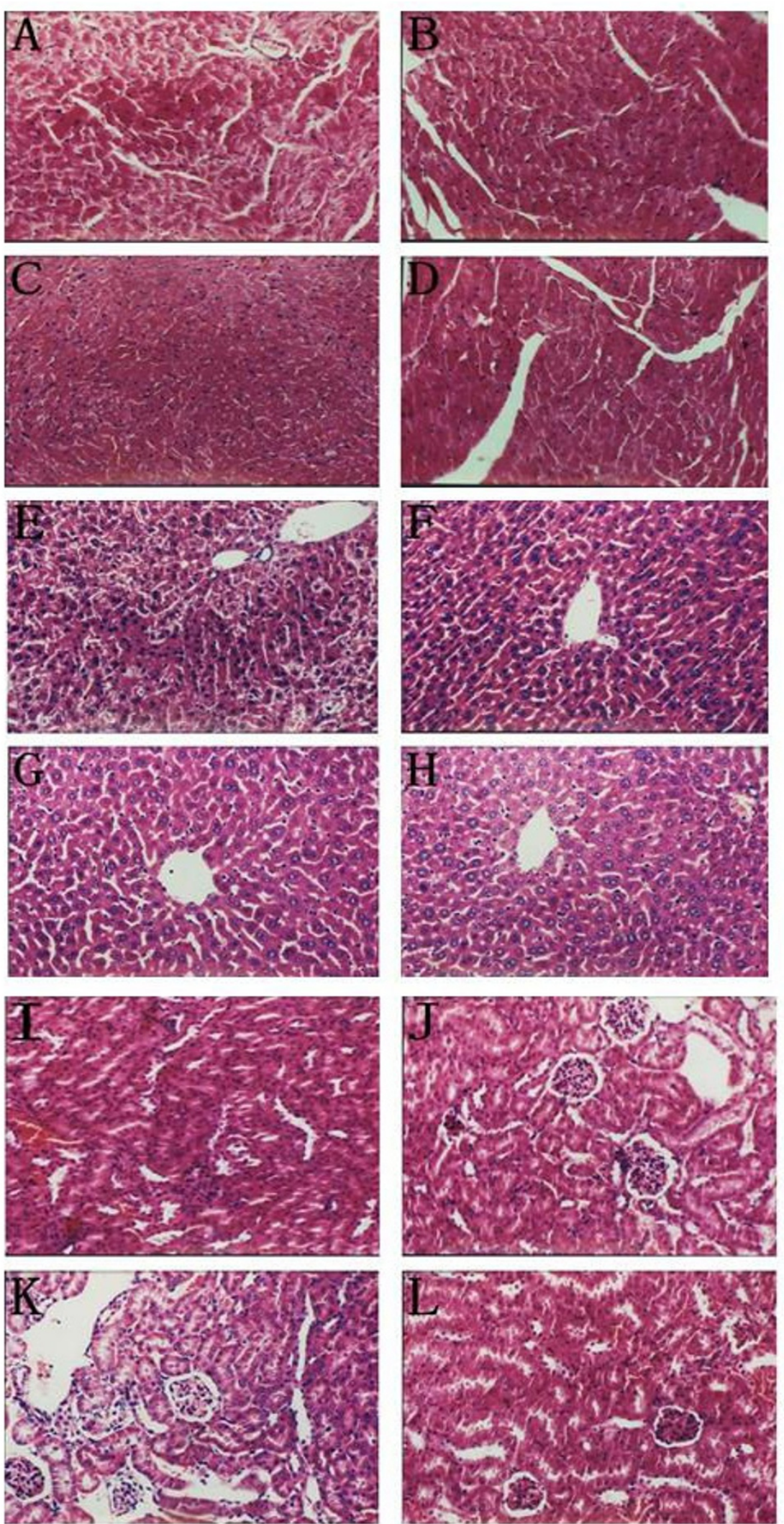

Figure 4 (see legend on next page) 
Figure 4 (see previous page)

The effects of YSL plus mid-dose doxorubicin on the internal organs of nude mice bearing human hepatocellular carcinoma BEL-7402 tumors. BEL-7402 cell ( $\times 107 /$ every mouse) was subcutaneously inoculated into the right back of every nude mouse. When the tumors had reached an average volume of $100 \mathrm{~mm}^{3}$, the tumor-bearing nude mice were randomly divided into different groups $(n=12)$. The mice were administrated i.p. 30 days. At the next day of the last administration, nude mice were sacrificed, and then put the heart, liver and kidney tissue into $10 \%$ formalin to fix and paraffin-embedded sections of each tissue sample were stained routinely with hematoxylin and eosin (HE). Figures 4A, 4B, 4C and 4D are heart samples. Figures 4E, 4F, 4G, and 4H are liver samples. Figures 4I, 4J, 4K and 4L are kidney samples. Figure 4A (Mid-dose doxorubicin): Myocardial cells are pultaceous and characterized by enhanced acidophilia. Figure 4B (YSL plus mid-dose doxorubicin): The transverse striation of myocardial cells is clear and with good repair. Figure 4C (YSL group) and Figure 4D (Saline group): There are no obvious abnormalities. Figure 4E (Mid-dose doxorubicin): There was enhanced acidophilia and interstitial edema. Figure 4F (YSL plus mid-dose doxorubicin): The structure of hepatic lobules is clear. Figure 4G (YSL group) and Figure 4H (Saline group): There are no obvious abnormalities. Figure 4I (Mid-dose doxorubicin): There are congestion of renal glomeruli and narrowed Bowman's space. Figure 4J (YSL plus mid-dose doxorubicin): Nephric tubules exhibit swelling. Figure 4K (YSL group) and Figure 4L (Saline group): There are no obvious abnormalities.

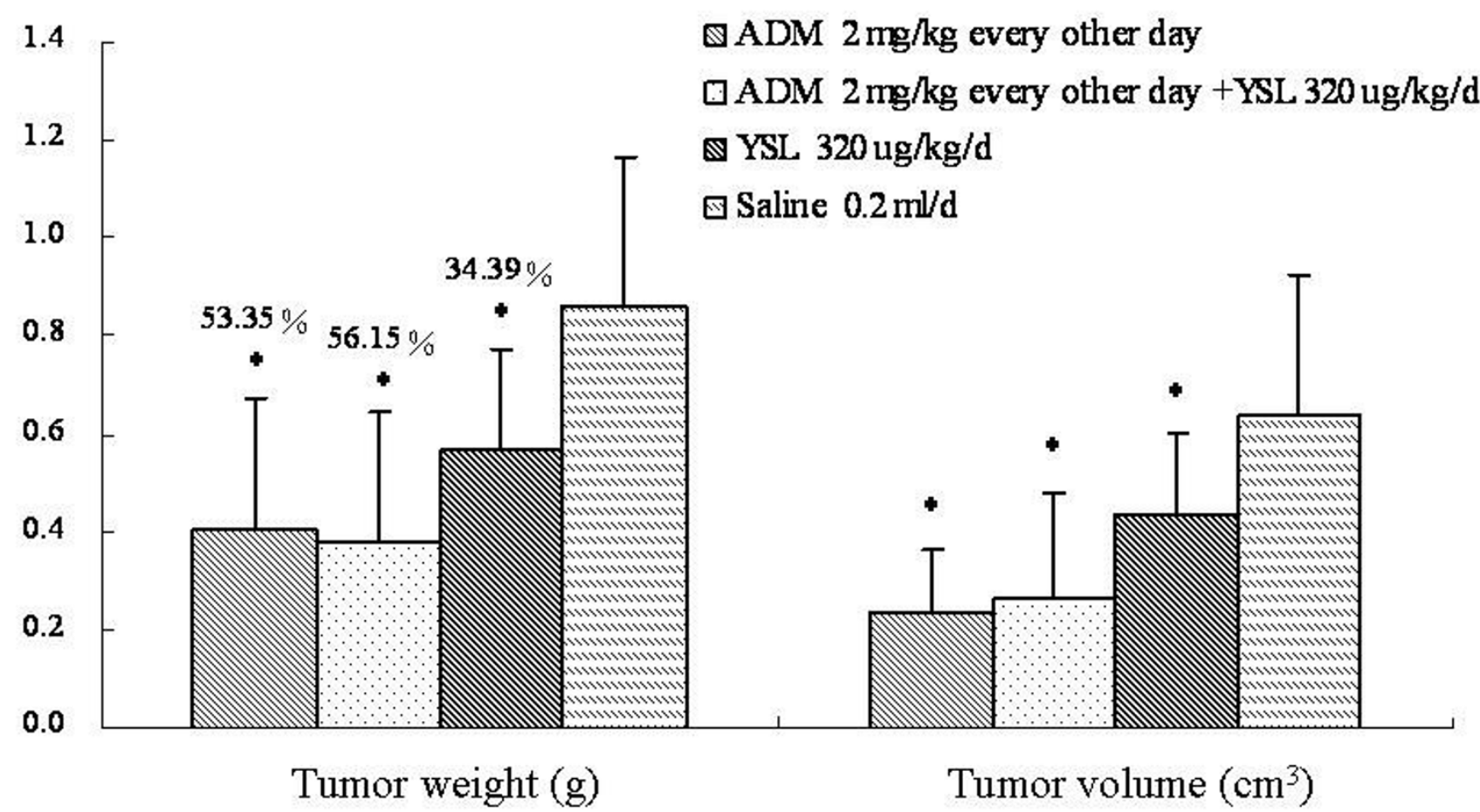

Figure 5

The effects of YSL plus mid-dose doxorubicin on tumor growth in nude mice bearing human hepatocellular carcinoma BEL-7402 tumors. BEL-7402 cell $(\mathrm{I} \times 107 /$ every mouse) was subcutaneously inoculated into the right back of every nude mouse. When the tumors had reached an average volume of $100 \mathrm{~mm}^{3}$, the tumor-bearing nude mice were randomly divided into different groups $(n=12)$. The mice were administrated i.p. 30 days. At the next day of the last administration, tumor tissue were stripped and three diameter which were vertical each other were measured. The tumor volume was calculated by $V\left(\mathrm{~cm}^{3}\right)=(\mathrm{I} / 6) \pi A B C$, and $A, B, C$ was three diameter of tumor. Then we weigh the tumor and calculated the tumor inhibition rate by tumor inhibition rate= (average weight of control group-average weight of experimental group)/average weight of control group $\times 100 \%$. All data were analyzed by one-way analysis of variance using SPSS. The difference between two groups was analyzed by the Student-Newman-Keuls test. Standard deviation was represented by error line. $* P<0.05$ as compared to the saline group. ADM, doxorubicin or adriamycin; YSL, tripeptide tyroserleutide. 


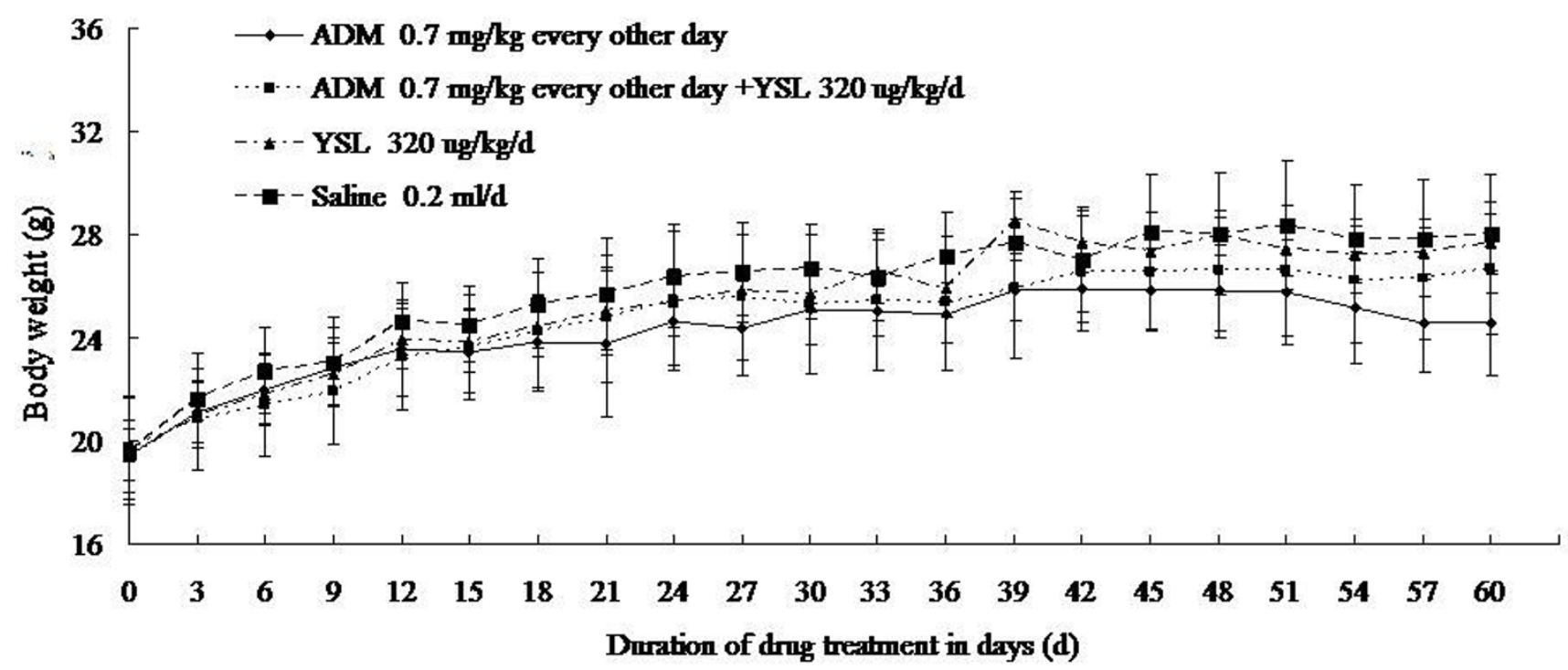

Figure 6

The effects of YSL plus low-dose doxorubicin on the body weight of nude mice bearing human hepatocellular carcinoma BEL-7402 tumors. BEL-7402 cell $\left(\mathrm{I} \times 10^{7} /\right.$ every mouse) was subcutaneously inoculated into the right back of every nude mouse. When the tumors had reached an average volume of $100 \mathrm{~mm}^{3}$, the tumor-bearing nude mice were randomly divided into different groups $(n=12)$. The mice were administrated i.p. 60 days. The body weights were weighing by electronic balance every three day. Standard deviation was represented by error line. ADM, doxorubicin or adriamycin; YSL, tripeptide tyroserleutide.

YSL in combination with mid-dose doxorubicin improves the survival status of nude mice bearing tumors and decreases doxorubicin-associated side effects such as weight loss, decreased body temperature, and reduced activity level. These data suggest that YSL plus doxorubicin both prolongs the survival time of nude mice bearing tumor and enhances their survival quality, which has important clinical implications.

Bone marrow depression is a common side effect of doxorubicin, and the influence on leukocyte counts is the most obvious. About $60-80 \%$ of patients have leukocyte depression after 10-15 days of doxorubicin therapy, and they recover by about day 21 of therapy [18]. YSL has strong protective effect on doxorubicin-induced leukocyte depression. In this study, YSL given in combination with mid-dose doxorubicin increased the number of leukocytes; thus, the inclusion of YSL in doxorubicin treatment regimens could reduce rate of infectious complications.

During therapy, doxorubicin can induce fatal cardiac toxicity, and doxorubicin-induced liver and kidney injury can be life threatening. Mid-dose doxorubicin can injure the heart, liver, and kidneys to varying degrees. We found that the degree of injury was reduced in the YSL combination group as compared to the doxorubicin group; that is, YSL seemed to prevent or repair doxorubicin-induced heart, liver, and kidney injury. The cardio- and hepatoprotective effects of YSL were especially obvious. In the YSL combination group the structure of cardiocyte was clear, hepatic lobules was complete, and the structure of the kidney cortical labyrinth and medullary ray was clear compared to the doxorubicin group.

The elevated doxorubicin levels in the blood conduce to reaching a high concentration in tumor tissue to increase the anti-tumor effects of doxorubicin [19]. However, some organs with abundant blood supply such as heart, liver and kidney will be damaged because of high doxorubicin concentration. In this study, we found that highand mid-dose doxorubicin had obvious anti-tumor effects on human hepatocellular carcinoma BEL-7402 cells, but doxorubicin also influenced the common status and basic vital signs of nude mice bearing tumors severely. Doxorubicin's side effects include hypo-leukocytosis, cardiac injury, liver and kidney toxicity, weight loss, and survival time decurtation. Compared with control group, lowdose doxorubicin induced less severe side effects, but had no significant inhibition on the growth of human hepatocellular carcinoma BEL-7402 cells in nude mice. Combination therapy with YSL significantly protected the mice from doxorubicin-induced side effects and increased the 


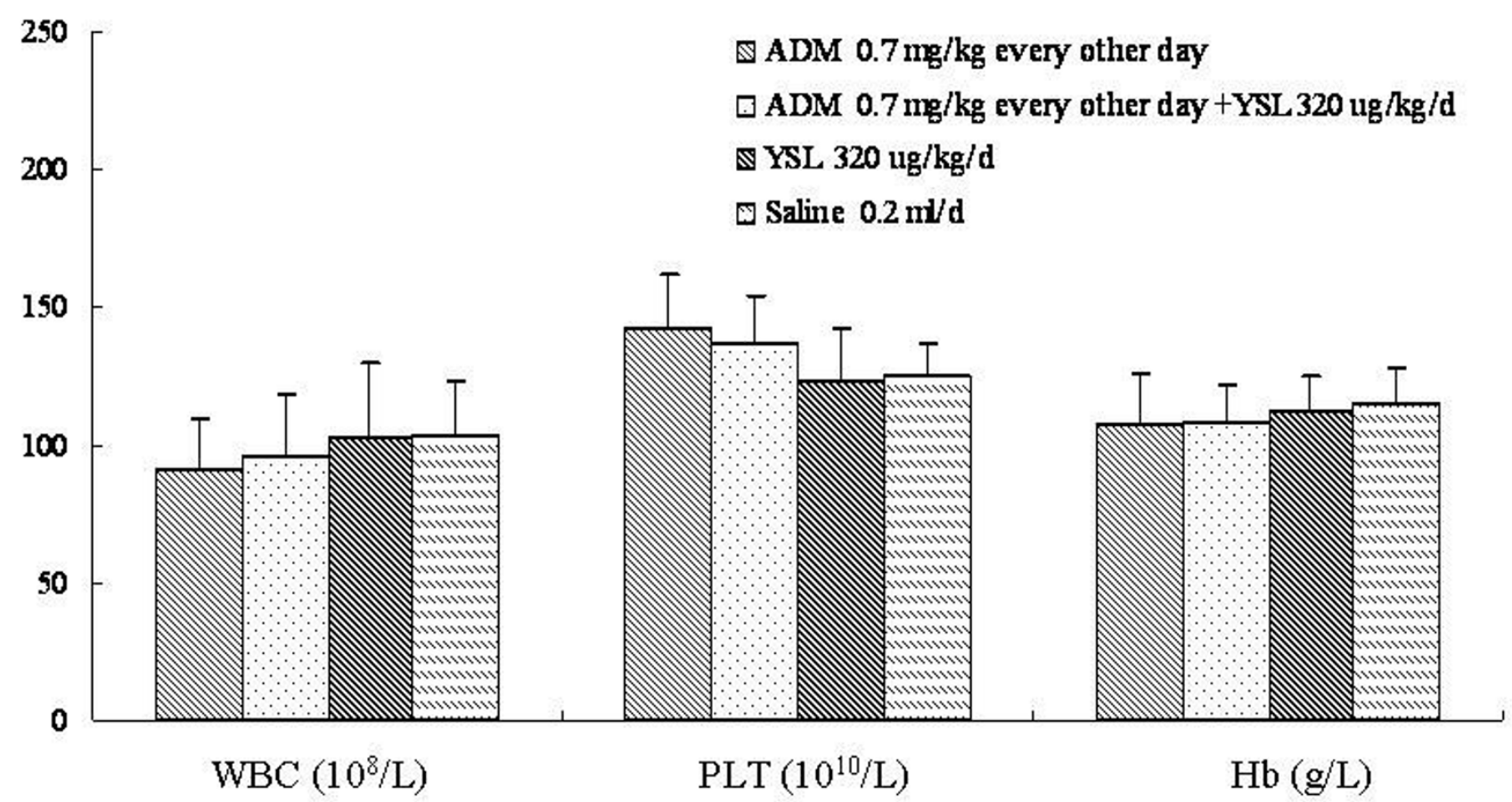

\section{Figure 7}

The effects of YSL combination with low dosage of doxorubicin on hematological indices of nude mice bearing human hepatocellular carcinoma BEL-7402 tumors. BEL-7402 cell ( $\times 107 /$ every mouse) was subcutaneously inoculated into the right back of every nude mouse. When the tumors had reached an average volume of $100 \mathrm{~mm}^{3}$, the tumor-bearing nude mice were randomly divided into different groups $(n=12)$. Drugs delivered by i.p. injection for 60 days successively. At the next day of the last administration, blood was obtained from angular vein of nude mice, then blood was anticoagulated by heparin, we detected hematoglobin level and counted PLT and WBC. All data were analyzed by one-way analysis of variance using SPSS. The difference between two groups was analyzed by the Student-Newman-Keuls test. Standard deviation was represented by error line. Statistical significance was set at $P<0.05$. ADM, doxorubicin or adriamycin; YSL, tripeptide tyroserleutide. WBC, white blood cells; PLT, platelets; Hb, hemoglobin.

anti-tumor effects of doxorubicin. YSL could induce the apoptosis of human hepatocellular carcinoma BEL-7402 cells [20]. And Xu et al found that tumor necrosis factorrelated apoptosis inducing ligand (TRAIL) could significantly increase the anti-tumor effects of doxorubicin through inducing the apoptosis of cancer cells [21]. So we think that the mechanisms enabling YSL to augment the cytotoxicity of doxorubicin may correlate well with the increased ability of doxorubicin when combined with YSL to induce apoptosis. Also YSL significantly increased the expression of PTEN in human hepatocellular carcinoma BEL-7402 cell. Over-expression of PTEN could increase the sensitivity to doxorubicin in human breast cancer MCF-7 cells [22]. The boosting of activity of doxorubicin when combined with YSL may result from the increased expression of PTEN to increase the sensitivity of BEL-7402 cells to doxorubicin.
In addition, our study observed the effects of co-administration of YSL and high-, mid-, and low-dose doxorubicin on nude mice bearing tumors. Although few side effects were recorded in low-dose doxorubicin group, there was no significant difference in tumor volumes and weights between the low-dose doxorubicin group and saline group. The tumor inhibition rate of YSL combination group was higher than that of the low-dose doxorubicin group, significantly different as compared to the saline group. YSL might enhance the efficacy of doxorubicin and have a dosage sparing effect which can reduce the dosage of doxorubicin to reduce its side-effects. Hence, it is possible that YSL can act as a new anti-tumor drug for the clinical development of combination with doxorubicin. Also YSL could reduce doxorubicin-induced side effects in combination with mid- and high-dose doxorubicin which produced significant anti-tumor effects. For example YSL prolonged the survival time of nude mice bearing tumor 


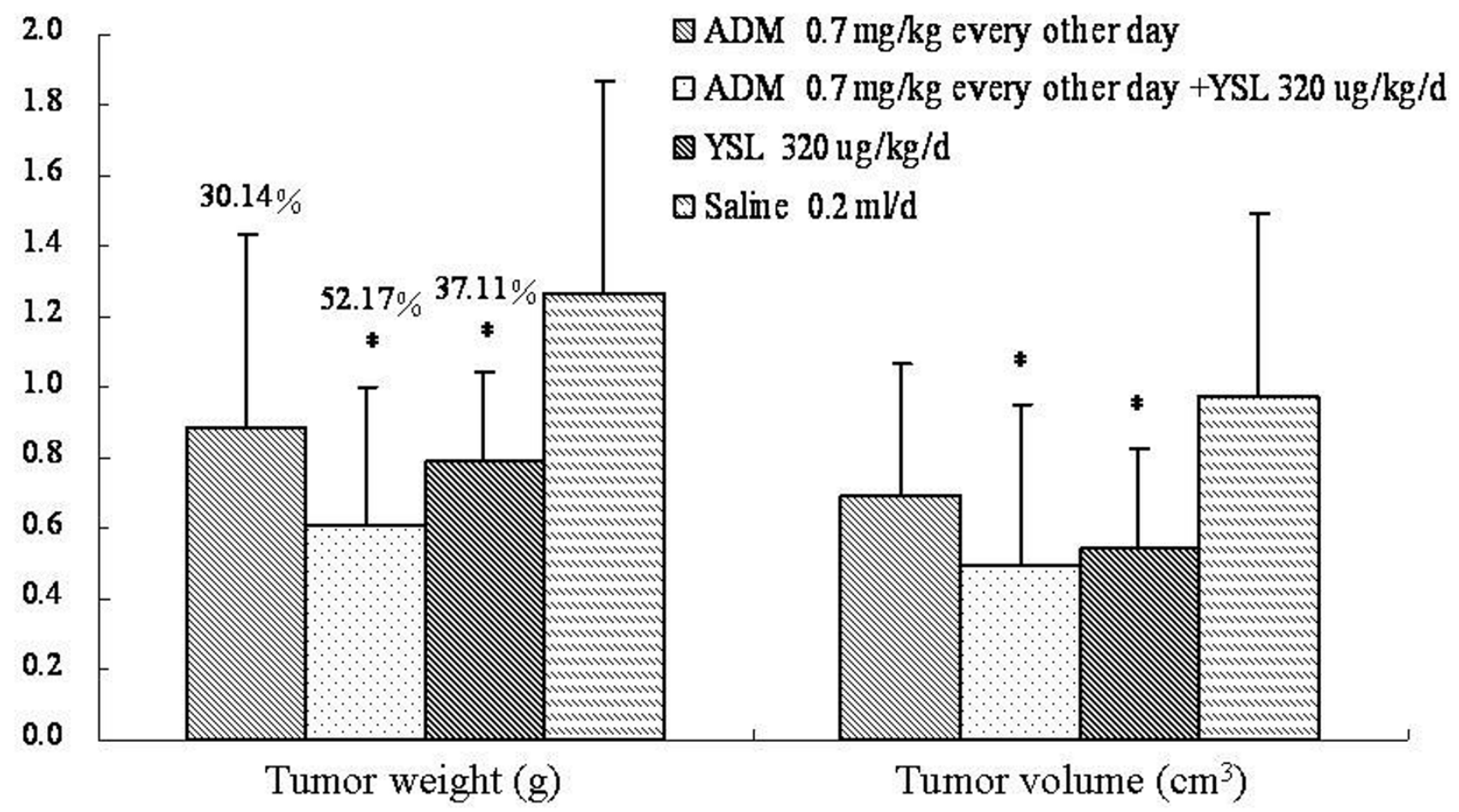

\section{Figure 8}

The effects of YSL plus low-dose doxorubicin on tumor growth in nude mice bearing human hepatocellular carcinoma BEL-7402 tumors. BEL-7402 cell ( 1 × 107/every mouse) was subcutaneously inoculated into the right back of every nude mouse. When the tumors had reached an average volume of $100 \mathrm{~mm}^{3}$, the tumor-bearing nude mice were randomly divided into different groups $(n=12)$. The mice were administrated i.p. 60 days. At the next day of the last administration, tumor tissue were stripped and three diameter which were vertical each other were measured. The tumor volume was calculated by $V\left(\mathrm{~cm}^{3}\right)=(1 / 6) \pi A B C$, and $A, B, C$ was three diameter of tumor. Then we weigh the tumor and calculated the tumor inhibition rate by tumor inhibition rate= (average weight of control group-average weight of experimental group)/average weight of control group $\times 100 \%$. All data were analyzed by one-way analysis of variance using SPSS. The difference between two groups was analyzed by the Student-Newman-Keuls test. Standard deviation was represented by error line. $* P<0.05$ as compared to the saline group. ADM, doxorubicin or adriamycin; YSL, tripeptide tyroserleutide.

and overcame the negative side effects of doxorubicin by protecting hematological and vital organ health. Doxorubicin can undergo redox cycling to produce reactive oxygen species. The oxidant-generating activity of doxorubicin is thought to be responsible for the side effects of the drug [23]. Further research needs to be carried out to test whether YSL would interfere with oxidantgenerating activity of doxorubicin to inhibit side effects of drug. Moreover, the mechanism accounting for sideeffects of doxorubicin mainly depends on its cytotoxicity. Further experiment is required to show that YSL could selectively induce apoptosis of human hepatocellular carcinoma cells but not normal cells from various organs.

\section{Conclusion}

In this study, the potential attenuation and synergistic effect of tripeptide tyroserleutide plus doxorubicin against human hepatocellular carcinoma in nude mice were investigated. Co-administration of YSL and low-, mid-, or high-dose doxorubicin respectively decreased chemotherapy-associated weight loss, leukocyte depression, and heart, liver, and kidney damage as compared to doxorubicin alone. Although there were no statistically significant differences in tumor weights between doxorubicin alone group and YSL plus doxorubicin group, YSL plus doxorubicin produced greater tumor inhibition rate than doxorubicin. The combination of YSL plus doxorubicin increased the sensitivity of tumors to chemotherapeutic drugs and reduces the side effects associated with doxorubicin chemotherapy.

\section{Abbreviations}

YSL: tyroserleutide; ADM: Doxorubicin or Adriamycin; IP: intraperitoneally; HE: hematoxylin and eosin; SD: stand- 
ard deviation; ANOVA: analysis of variance; ASCO: American Society of Clinical Oncology; TRAIL: tumor necrosis factor-related apoptosis inducing ligand; WBC: white blood cells; PLT: platelets; Hb: hemoglobin.

\section{Competing interests}

The authors declare that they have no competing interests.

\section{Authors' contributions}

ZZF and CLJ contributed to perform the animal study. LR designed the study, JJ contributed to the statistical analysis. LY was in charge of histopathology examinations. XQ and ZCL contributed to the data collection. WL was in charge of blood counts. WS was in charge of the tumors' collection. YZ wrote the manuscript.

\section{All authors read and approved the manuscript.}

\section{Acknowledgements}

This work was supported by Grants for the National High Technology Research and Development Program of China ("863" Program, China) (2004AA2Z3 I70, 2005AA2Z3D40) and the National Basic Research Program ( 973 Program, China) and by an important project grant (03007) from the Department of Education of China.

\section{References}

I. Yang JL, Wang Q, Wu ZY, Li X, Wang YP: The effect of genistein on the proliferation of human hepatoceUular carcinoma cell SMMC-772I. Chinese Journal of Hepatology 2006, 08:622-623.

2. Ganey PE, Carter LS, Mueller RA, Thurman RG: Doxorubicin toxicity in perfused rat heart. Decreased cell death at low oxygen tension. Circ Res 1991, 68:1610-1613.

3. Yao Z, Lu R, Jia J, Zhao P, Yang J, Zheng M, Lu J, Jin M, Yang H, Gao $\mathrm{W}$ : The effect of tripeptide tyroserleutide (YSL) on animal models of hepatocarcinoma. Peptides 2006, 27:I I67-II72.

4. Lu R, Jia J, Bao L, Fu Z, Li G, Wang S, Wang Z, Jin M, Gao W, Yao Z: Experimental study of the inhibition of human hepatocarcinoma BEL-7402 cells by the tripeptide tyroserleutide(YSL). Cancer Chemother Pharmacol 2006, 57:248-256.

5. Han R: Anticancer drugs research and exoeriment technology Beijing: Beijing Medical University and Peking Union Medical College association publishing company; 1997.

6. Volm M, Faufmann M, MAttern J, Wayss K: Sensitivity tests of tumors to cytostatic agents. I. Comparative investigations on transplanted tumors in vivo and in vitro. $Z$ Krebsforsch Klin Onkol Cancer Res Clin Oncol 1975, 83:85-96.

7. Igawa M, Kadena $\mathrm{H}$, Ueda M, Usui T: Association between patient characteristics and treatment history, and toxicity associated with methotrexate, vinblastine, adriamycin and cisplatin (M-VAC) for advanced urothelial cancer. $\mathrm{Br} J$ Urol 1994 , 73:263-267.

8. Zeidán Q, Strauss M, Porras N, Anselmi G: Differential long-term subcellular responses in heart and liver to adriamycin stress. Exogenous L-carnitine cardiac and hepatic protection. J Submicrosc Cytol Pathol 2002, 34:3 I5-32I.

9. Di Donato $A$, Ghiggeri GM, Di Duca M, Jivotenko E, Acinni R, Campolo J, Ginevri F, Gusmano R: Lysyl oxidase expression and collagen cross-linking during chronic adriamycin nephropathy. Nephron 1997, 76: 192-200.

10. Singal PK, Deally CM, Weinberg LE: Subcellular effects of adriamycin in the heart: a concise review. J Mol Cell Cardiol 1987, 19:817-828.

II. Lefrak EA, Pitha J, Rosenheim S, Gottlieb JA: A clinicopathologic analysis of adriamycin cardiotoxicity. Cancer 1973, 32:302-314.

12. Weil-Hillman G, Uckun FM, Manske JM, Vallera DA: Combined immunochemotherapy of human solid tumors in nude mice. Cancer Res 1987, 47:579-585.
13. Boehmer S, Luszczynska A, Schwarzer R: Coping and quality of life after tumor surgery: Personal and social resources promote different domains of quality of life. Anxiety Stress Coping 2007, 20:6I-75.

14. Schmitt M, Harbeck N, Daidone MG, Brynner N, Duffy MJ, Foekens JA, Sweep FC: Identification, validation, and clinical implementation of tumor-associated biomarkers to improve therapy concepts, survival, and quality of life of cancer patients: tasks of the Receptor and Biomarker Group of the European Organization for Research and Treatment of Cancer. Int J Oncol 2004, 25: I 397- I 406.

15. Poulakis V, Skriapas K, de Vries R, Dillenburg W, Ferakis N, Witzsch $U$, Becht E: Quality of life after laparoscopic and open retroperitoneal lymph node dissection in clinical Stage I nonseminomatous germ cell tumor: a comparison study. Urology 2006, 68: $154-160$.

16. Bampoe J, Ritvo P, Bernstein M: Quality of life in patients with brain tumor: what's relevant in our quest for therapeutic efficacy. Neurosurg Focus 1998, 4:e6.

17. Shaw EG, Rosdhal R, D'Agostino RB Jr, Lovato J, Naughton MJ, Robbins ME, Rapp SR: Phase II study of donepezil in irradiated brain tumor patients: effect on cognitive function, mood, and quality of life. J Clin Oncol 2006, 24:|4|5-|420.

18. Liao ZJ: Modern oncotherapy De material medica Xi'an: World Publishing Corporation; 2002.

19. Stallard S, Morrison JG, George WD, Kaye SB: Distribution of doxorubicin to normal breast and tumour tissue in patients undergoing mastectomy. Cancer Chemother Pharmacol 1990, 25:286-290.

20. Lu R, Jia J, Bao L, Fu Z, Li G, Wang S, Wang Z, Jin M, Gao W, Yao Z: Experimental study of the inhibition of human hepatocellular carcinoma Bel7402 cells by the tripeptide tyroserleutide(YSL). Cancer Chemother Pharmacol 2006, 57:248-256.

21. Xu LH, Deng CS, Zhu YQ, Liu SQ, Liu DZ: Synergistic antitumor effect of TRAIL and doxorubicin on colon cancer cell line SW480. World J Gastroenterol 2003, 9:124I-I245.

22. Lin XY, Zhou GY, Song YH, Gao P, Sun YL: PTEN inhibits cell growth and increases chemosensitivity to doxorubicin of human breast cancer cells. Chinese J General Surgery 2005, 20:240-242.

23. Hoke EM, Maylock CA, Shacter E: Desferal inhibits breast tumor growth and does not interfere with the tumoricidal activity of doxorubicin. Free Radic Biol Med 2005, 39:403-4II.

\section{Pre-publication history}

The pre-publication history for this paper can be accessed here:

\section{http://www.biomedcentral.com/1471-2407/8/342/pre} pub

\footnotetext{
Publish with Biomed Central and every scientist can read your work free of charge

"BioMed Central will be the most significant development for disseminating the results of biomedical research in our lifetime. "

Sir Paul Nurse, Cancer Research UK

Your research papers will be:

- available free of charge to the entire biomedical community

- peer reviewed and published immediately upon acceptance

- cited in PubMed and archived on PubMed Central

- yours - you keep the copyright

Submit your manuscript here:

http://www.biomedcentral.com/info/publishing_adv.asp

BioMedcentral
} 